\title{
Variations
}

Variations

Revue internationale de théorie critique

$24 \mid 2021$

Echos

\section{Le post-scriptum comme préface : théoriser le contrôle après Deleuze}

Jason Read

Traducteur : David Buxton

(2) OpenEdition

Journals

Édition électronique

URL : https://journals.openedition.org/variations/2048

DOI : 10.4000/variations. 2048

ISSN : 1968-3960

Éditeur

Les amis de Variations

\section{Référence électronique}

Jason Read, «Le post-scriptum comme préface : théoriser le contrôle après Deleuze », Variations [En

ligne], 24 | 2021, mis en ligne le 03 juillet 2021, consulté le 07 juillet 2021. URL : http://

journals.openedition.org/variations/2048; DOI : https://doi.org/10.4000/variations.2048

Ce document a été généré automatiquement le 7 juillet 2021.

Les ami•e•s de Variations 


\title{
Le post-scriptum comme préface : théoriser le contrôle après Deleuze
}

\author{
Jason Read \\ Traduction : David Buxton
}

\section{NOTE DE L'ÉDITEUR}

Publication originale dans Coils of the Serpent, $n^{\circ} 6,2020$.

1 «Post-scriptum sur les sociétés de contrôle» de Gilles Deleuze fonctionne comme l'index d'un changement d'époque. Le texte, qui commence avec une évocation du passé, situant la théorie foucaldienne du pouvoir disciplinaire au $19^{\mathrm{e}}$ siècle, a été lu comme une théorisation du présent, des déplacements du pouvoir à la fin du $20^{\mathrm{e}}$ siècle. Quel est son legs? Son avenir? Il me semble que, trente ans après sa publication, on peut lui poser deux séries de questions. D'abord, quid du contrôle aujourd'hui? Comment, en tant que technique du pouvoir, s'est-il transformé ? Est-il possible d'affirmer que nous vivons dans une nouvelle ère de pouvoir, distincte de la discipline et du contrôle? En second lieu, de quels outils et de quelles ressources disposons-nous pour théoriser le contrôle aujourd'hui ? Comment cette théorie s'est-elle développée depuis Deleuze? Sur ce dernier point, il faut noter que son court essai est aussi fragmentaire qu'il est prescient, construisant ce qui est presque une théorie impressionniste à travers quelques références à Foucault, à Burroughs, à la sciencefiction, et à l'entreprise moderne. Il reste donc beaucoup à faire pour en élaborer une théorie qui irait au-delà d'une description du présent, afin de saisir les mutations du contrôle au $21^{\mathrm{e}}$ siècle. Les deux séries de questions, l'une traitant l'objet et l'autre le concept, constituent moins des lignes d'enquête différentes que des interrogations croisées sur son legs.

2 Pour comprendre la transformation du contrôle, il faut s'arrêter sur l'historicisation d'un tel concept. Deleuze situe le contrôle comme quelque chose qui déplace la discipline, de même que Foucault affirme que celle-ci déplace la souveraineté en tant 
que forme de pouvoir. La discipline opère dans des lieux nécessairement fermés l'école, la prison, la caserne - comme une forme de pouvoir qui s'exerce dans l'observation et dans l'enfermement. Passer d'un lieu à un autre, c'est traverser des espaces distincts, mais similaires. Par contraste, les espaces de contrôle sont ouverts et en état de flux constant. La vie qui passe de l'école à l'armée puis au travail est remplacée par une vie d'éducation continue, de travail flexible et de surveillance distribuée. Il y a, cependant, de la continuité dans cette discontinuité. L'un des arguments, souvent commenté, chez Foucault à l'égard de la discipline est que le pouvoir est d'autant plus persuasif qu'il devient moins matériel, moins focalisé sur l'infliction de la douleur physique ou même de l'enfermement, et agissant à travers des perceptions et des idées plutôt que directement sur des corps. Le panoptique est plus fort que les verrous, plus sûr que la cellule, car il ne cherche pas à contenir le corps, mais agit sur l'esprit. La structure de visibilité (l'exposition des confinés), et la structure d'invisibilité (l'incapacité à savoir quand on est surveillé) produisent une connaissance de soi, une conscience, une âme même. Comme le dit Foucault, décrivant cette relation : "L'âme, effet et instrument d'une anatomie politique ; l'âme, prison du corps (1975 : 38). » Autant le contrôle se présente en principe comme une rupture avec la discipline sur le plan de l'organisation spatiale du pouvoir, remplaçant l'enfermement et les espaces discrets avec la régulation des flux de populations et des modulations continues du pouvoir, autant il poursuit la tendance vers l'internalisation et l'incorporation du pouvoir. Celui-ci agit de moins en moins sur le corps, sur son enfermement, et de plus en plus sur les pensées et idées régissant son activité. Foucault définit le pouvoir comme la conduite de la conduite, et le contrôle prolonge cette trajectoire, à mesure que toute action se renvoie aux conditions de conduites.

Deleuze donne deux exemples de cette incorporation accrue du pouvoir dans les sociétés de contrôle. D'abord, et de façon proéminente, la dette. Comme le dit Deleuze, «l'homme n'est plus l'homme enfermé, mais l'homme endetté ». Ensuite, en guise de remarque concluante, il écrit: "Beaucoup de jeunes gens réclament étrangement d'être "motivés", ils redemandent des stages et de la formation permanente. » Ces deux affirmations définissent le nadir et le zénith du contrôle comme forme d'assujettissement. À un pôle se trouve la dette, qui fonctionne comme une forme d'enfermement, une prison d'autant plus efficace qu'elle n'agit pas directement sur des corps, ni même sur des esprits, mais enferme par l'imposition d'une matrice de calcul et de risque individualisé de nos actions et décisions. La dette emprisonne sans prison, sans tour de surveillance, faisant de nous nos propres gardiens contrôlant les risques, les coûts et les bénéfices de nos vies. À l'autre pôle se trouve la motivation, qui se présente comme une impulsion interne. Dans L'Anti-ÆEdipe, Deleuze et Guattari déclarent que la question centrale de la politique reste celle posée autrefois par Spinoza: "pourquoi les hommes combattent-ils pour leur servitude comme s'il s'agissait de leur salut?» De nos jours, il est moins question de se battre et de mourir pour la gloire d'un homme que de démêler la motivation de l'assujettissement. Comme l'écrit Deleuze, « c'est [aux jeunes gens] de découvrir ce à quoi on les fait servir, comme leurs aînés ont découvert non sans peine la finalité des disciplines ». Dans la mesure où le concept de contrôle déplace celui de discipline, il le fait de telle manière que ses contraintes, ses intérêts et ses motivations ne sont pas facilement discernés. À la suite des remarques de Deleuze sur Foucault, on peut affirmer que le contrôle est plus difficile à saisir, car nous sommes toujours sous son emprise. Dans ce cas, la chouette de Minerve (Hegel) n'a pas encore pris son envol. 
4 À l'égard de la dette comme forme de contrôle, l'exemple paradigmatique depuis Deleuze serait la dette estudiantine. Son essai est étrangement prescient, du moins en ce qui concerne les États-Unis, quant à la condition changeante des étudiants, moins sujets à l'imposition d'un appareil disciplinaire singulier qu'ils ne subissent les formes de pouvoir chevauchées et dispersées de la famille, du travail précaire, de la formation continue, et de la dette. L'étudiant est quelqu'un qui vit avec ou sous l'autorité de ses parents, qui travaille un peu, et qui finance son éducation en s'endettant. Il est contraint par le passé, le présent et le futur comme autant de logiques poreuses de pouvoir et de contrôle. La dette, c'est le futur agissant sur le présent. Plus on est obligé de financer ses études par la dette, plus on est poussé à considérer cette éducation comme un investissement plutôt qu'une exploration. Logiquement, la figure de l'étudiant sous-tend le second cas évoqué par Deleuze, non quelqu'un contraint par la dette, mais qui s'engage volontairement dans un processus continu de formation. Maurizio Lazzarato est le principal penseur contemporain qui a contribué à élaborer une théorie du contrôle après Deleuze, exprimant l'idée générale de contrôle comme nouveau régime de pouvoir, et la fonction spécifique de la dette comme forme de contrôle. À l'égard de cette dernière, Lazzarato affirme que le rapport entre enfermement et motivation chez Deleuze doit se comprendre comme une production particulière de la subjectivité. Comme l'écrit Lazzarato :

"L'économie de la dette se caractérise alors par un double élargissement de l'exploitation de la subjectivité : extensive (puisqu'il ne concerne pas que l'emploi industriel ou de services, mais toute activité et condition), et intensif (puisqu'il concerne le rapport à soi, sous la forme d'entrepreneur de soi à la fois responsable de "son" capital et coupable de sa mauvaise gestion, dont le paradigme est le "chômeur").» (2012: 43)

5 L'expansion intensive de l'exploitation de la subjectivité est préfigurée par le jeune Marx dans ses notes critiques sur James Mill. Ce qui frappe dans ce texte, c'est que la dette n'est pas vue comme aliénation de l'humanité à travers l'argent (où ce qui est spécifiquement humain est perdu au calcul abstrait), mais représente plutôt la transformation de l'individualité en incarnation vivante de l'esprit d'argent. La dette transforme donc chaque aspect de la personnalité, les habitudes, les histoires, et les désirs en quelque chose de calculable. Comme l'écrit Marx :
«Au sein de la relation du crédit, ce n'est pas que l'argent soit transcendé en l'homme, mais que l'homme lui-même soit transformé en argent, ou que l'argent soit incorporé en lui. L'individualité humaine, la moralité humaine elle-même, est devenue à la fois un objet du commerce, et de la matière dans laquelle l'argent existe. Au lieu de la monnaie ou du papier, c'est ma propre existence, ma chair et mon sang, ma vertu et mon importance sociale qui constituent la forme matérielle, corporelle de l'esprit d'argent. Le crédit ne résout plus la valeur de l'argent dans de l'argent, mais dans la chair humaine et dans le cœur humain. Telle est la mesure à laquelle tout progrès et toute inconsistance au sein d'un système faux sont l'extrême rétrogression et l'extrême conséquence de la vilenie ${ }^{1}$. » (1932 (1844)).

Ce que Lazzarato souligne, c'est que cet assujettissement revêt une dimension morale. La dette et la capacité ou non à payer prennent un langage spécifiquement moral de culpabilité et de responsabilité. C'est là une inversion de ce qu'avance Nietzche: ce n'est pas que la moralité se dérive d'une économie de la dette, mais que celle-ci soit moralisée de fond en comble. Ceux qui ne peuvent payer leurs dettes sont non seulement perçus comme de mauvais agents économiques, mais surtout comme des irresponsables. La dette fonctionne comme une prison, un mode d'enfermement, en 
forçant les gens à calculer les risques de leurs actions d'une part, et en les tenant pour responsables de l'autre.

7 Cela dit, l'insistance de Lazzarato sur la nature moralisante de la dette oublie les mécanismes par lesquels la dette est émise et collectée. Le tableau dressé par Marx d'une incorporation personnelle n'est plus en phase avec la façon dont la dette a évolué au $20^{\circ}$ siècle (McClanahan, 2017, 79). La dette est devenue non seulement nécessaire pour l'accession à la propriété et le financement des études supérieures, mais aussi pour compenser le déclin des salaires. Plus précisément, cela correspond étroitement à la transformation générale de l'individuation que Deleuze associe au contrôle. Alors que la discipline se situe à l'intersection des masses et des individus (divisant les êtres humains en groupes discrets, prisonniers, soldats, ouvriers, etc., tout en les individualisant à travers des dossiers, des fiches, etc.), la dette ne donne pas lieu à une collectivité, à une masse, car les endettés ne constituent pas un groupe perceptible. Plutôt, ce sont des inconnus, les uns aux autres, et à eux-mêmes. La dette ne traite pas la personne en tant que telle, mais sélectionne les faits pertinents (antécédents de crédit, etc.), croisés avec d'autres données pour construire des modèles statistiques. Comme le dit Deleuze : «Les individus sont devenus des "dividuels", et les masses, des échantillons, des données, des marchés ou des "banques".» Ce qui définit la dette comme système de contrôle, c'est moins la moralisation et l'individualisation que l'incapacité à reconnaître la dette, soit comme une condition collective, soit comme un destin individuel. À la différence du travail, qui implique nécessairement une socialisation minime sur le lieu de celui-ci, la dette est le fait d'une communauté totalement invisible. De plus, pour l'individu, dans la mesure où la dette est liée aux dépenses non évitables (logement, santé, éducation), elle apparait moins comme une promesse ou un contrat que comme une réalité inéluctable de la vie.

Empruntant la distinction faite par Deleuze et Guattari dans Mille Plateaux, on pourrait affirmer que la dette fonctionne plutôt à la manière d'un «asservissement machinique » que d'un «assujettissement social ». Deleuze et Guattari définissent ces termes ainsi :

«Il y a asservissement lorsque les hommes sont eux-mêmes pièces constituantes d'une machine qu'ils composent entre eux et avec d'autres (bêtes, outils), sous le contrôle et la direction d'une unité supérieure. Il y a assujettissement lorsque l'unité supérieure constitue l'homme comme un sujet qui se reporte à un objet devenu extérieur, que cet objet soit lui-même une bête, un outil, ou même une machine ... » (1980 : 570-71).

9 À l'égard de la dette comme forme de contrôle, les pièces constituantes sont les paquets de données qui composent les "dividuels » et les banques de données, toujours en dessous de l'individu. Culpabilisant et angoissant, le discours moralisateur sur la dette, sur le risque personnel, et sur la demande catégorique de remboursement existe sur le plan de l'assujettissement social : de l'idéologie, des récits et des valeurs qui s'adressent aux individus en tant que travailleurs ou citoyens responsables. De cette façon, l'extraction de la richesse à travers la dette constitue la radicalisation d'une tendance déjà à l'œuvre dans le processus du travail. Dans l'exploitation de la force de travail, on peut faire une distinction entre l'asservissement machinique du travail, d'une part, et l'assujettissement social de l'ouvrier individuel, de l'autre. Comme l'écrit Lazzarato :

«Le capital ne fait pas que simplement extorquer une extension du temps de travail (la différence entre le temps humain rémunéré et le temps humain passé sur le lieu de travail), il instaure un processus qui exploite la différence entre assujettissement 
et asservissement. Car si l'assujettissement subjectif - l'aliénation sociale inhérente à un travail particulier ou à n'importe quelle fonction sociale (ouvrier, chômeur, enseignant, etc.) - est toujours assignable et mesurable (le paiement approprié à son poste, le salaire approprié à une fonction sociale), la part d'asservissement machinique constituant la production réelle n'est jamais assignable ni mesurable. » (2014: 45 , je traduis)

On pourrait ajouter que la stratégie du capital est de masquer la dimension machinique, collective du travail autant que faire se peut. L'invisibilité de la dette n'est qu'une version extrême de la tendance à masquer la dimension collective du travail. Comme l'écrivent Michael Hardt et Antonio Negri, « la productivité est de plus en plus cachée à mesure que les divisions entre temps de travail et temps de vie deviennent de plus en plus floues ». Les lieux de travail collectifs (usines, centres d'appel) sont remplacés par l'individu enchaîné à son bureau; des industries entières, telles que le transport et le logement, sont transformées en relations entre individus et machines. Dans chaque cas, ce qui est masqué et gommé, ce sont les conditions forcément collectives régissant l'individu présent sur son lieu de travail ou connecté à une plateforme en ligne. La dette masque les conditions collectives du travail, du logement, de l'éducation et de la santé, alors que les conditions du travail d'aujourd'hui masquent la performance collective. Comme le dit Deleuze du capitalisme dans l'âge du contrôle : "Ce qu'il veut vendre, c'est des services, et ce qu'il veut acheter, ce sont des actions. » La production, y compris la production des conditions de production, est forcément masquée.

11 En dépit de la tendance à considérer l'assujettissement social comme un déplacement de l'enfermement machinique, la trajectoire entre les deux est plus rectiligne que linéaire. D'après Deleuze et Guattari : «On dirait aussi bien qu'un peu de subjectivation nous éloignait de l'asservissement machinique, mais que beaucoup nous y ramène (1980 : 572). » Pour comprendre cette progression, il faut aussi comprendre la relation entre la forme d'assujettissement et la technologie. Dans Mille Plateaux, Deleuze et Guattari identifient la télévision comme une intersection entre le sujet et l'asservissement :

«Par exemple : on est assujetti à la télé pour autant qu'on en fait usage et qu'on consomme, dans cette situation très particulière d'un sujet d'énoncé qui se prend plus ou moins pour sujet d'énonciation ("vous, chers téléspectateurs, qui faites la télé...") ; la machine technique est le médium entre deux sujets. Mais, on est asservi par la télé comme machine humaine pour autant que les téléspectateurs sont, non plus des consommateurs ou des usagers, ni même des sujets censés la "fabriquer" mais des pièces composantes intrinsèques, des "entrées" et des "sorties", des feedback ou des récurrences, qui appartiennent à la machine et non plus à la manière de la produire ou de s'en servir. » $(1980: 573)$

12 La relation qu'établissent Deleuze et Guattari entre asservissement et assujettissement à l'égard de la télévision, où la réduction accrue des êtres humains à des pièces constituantes de la machine, aux "entrées» et aux «sorties", rend possible une expansion de la personnalisation et de l'assujettissement. Cette relation n'a fait que s'accélérer avec les technologies du $21^{\mathrm{e}}$ siècle naissant. Le développement d'Internet, et notamment des réseaux sociaux, a permis une montée de l'assujettissement, avec les pages dédiées à ses goûts particuliers, ses inclinations, ses humeurs, appuyées sur les couches invisibles d'enfermement machinique, alors que toute recherche et toute activité en ligne se transforment en données.

Deleuze situe la transition de la discipline au contrôle le long d'une transformation autant de la machinerie et de la technologie que des techniques de pouvoir et 
d'assujettissement. Le pouvoir disciplinaire est associé à la thermodynamique de la révolution industrielle, tandis que le contrôle est lié à l'informatique et aux technologies de l'information. Lazzarato affine cette périodisation en soutenant que le contrôle se fonde non seulement sur les flux d'information, mais aussi sur toute technologie qui fonctionne à travers des espaces et des temporalités dispersés. Le déclin des espaces enfermés propres à la discipline fait en sorte que le contrôle opère sur des foules disséminées dans l'espace et dans le temps, autrement dit, le public. En identifiant le contrôle avec le public, et avec les technologies de la radio, du cinéma, de la télévision, et d'Internet, Lazzarato fait deux pas en arrière au $19^{\mathrm{e}}$ siècle pour mieux aller de l'avant au $21^{\text {e }}$. Les pas en arrière sont d'ordre historique et théorique. D'abord, Lazzarato incorpore dans son analyse la presse, la radio et la télévision, technologies qui opéraient déjà à travers des espaces ouverts lors de l'âge de l'enfermement disciplinaire. Ces technologies sont absentes de l'histoire foucaldienne de la discipline, et ne figurent que de manière oblique dans l'essai de Deleuze. Les théoriciens du pouvoir et les théoriciens des médias œuvrent souvent en s'ignorant mutuellement, quand ils ne se trouvent pas en opposition totale ${ }^{2}$. Ensuite, Lazzarato revient en arrière également sur le plan théorique, se tournant vers la théorie des publics de Gabriel Tarde. Comme il l'écrit :

« Tarde saisit à leur naissance trois phénomènes qui vont caractériser les sociétés de contrôle et leur déploiement massif à partir de la seconde moitié du $20^{\mathrm{e}}$ siècle : 1) l'émergence de la coopération entre cerveaux et son fonctionnement par flux et par réseaux, networks et patchwork, 2) l'essor des dispositifs technologiques d'action à distance qui doublent et amplifient la puissance de l'action à distance des monades: télégraphe, téléphone, cinéma, télévision, Internet, 3) les processus de subjectivation et d'assujettissement correspondants ; la formation des publics, c'est-à-dire la constitution de l'être ensemble qui a lieu dans le temps. »

Si le contrôle se définit par des formes de pouvoir qui opèrent nécessairement à travers des espaces dispersés, alors ses outils sont non seulement la dette, la surveillance distribuée, et l'entreprise décentrée, mais aussi les technologies qui agissent sur l'imagination, l'affect et le désir, façonnant ainsi les conditions d'actions possibles. Selon Lazzarato, le contrôle doit être pensé comme une forme de pouvoir qui agit sur la possibilité même d'action. Alors que le panoptique contrôlait le prisonnier en créant chez lui la conscience d'être surveillé, encadrant toute action par ses répercussions potentielles, les formes de contrôle médiatisées agissent aussi sur les actions en façonnant un sens de ce qui est possible ou désirable. Le contrôle fait partie de la « noopolitique ", qui opère non sur les actions, mais sur les pensées portant sur la possibilité d'action (Lazzarato, 2004). La noo-politique est donc le point culminant d'une trajectoire qui passe par la discipline et par le contrôle; le pouvoir devient d'autant plus persuasif, d'autant plus efficace à mesure qu'il devient plus abstrait.

Comme l'affirme Yves Citton, les technologies qui disséminent des idées, des affects et des attentions à travers des espaces divers ne peuvent fonctionner sans une technologie plus ancienne, à savoir le récit. Les formes narratives constituent des moyens puissants pour façonner l'attention, pour modeler les affects et les désirs. Il existe un récit de responsabilité personnelle qui rend effective la dette, même si les relations réelles de celle-ci ont noyé l'individu dans une mer de profils « dividuels » et de banques agrégées. Autrement dit, autant l'asservissement machinique opère sur des individus réduits à des flux d'information, à des données regroupées dans les banques et les centres dédiés, autant l'assujettissement social du contrôle engage les individus de plus en plus à travers des fantasmes concernant la capacité d'agir et la 
responsabilité. Pour Citton, la noo-politique implique nécessairement une mythocratie, qui s'appuie non pas tant sur les mythes, mais sur les récits et les scénarios qui circulent entre la culture populaire et la politique. Ce qui occupe l'esprit, ce qui exige de l'attention et nous tient en haleine, ce sont des récits ${ }^{3}$. Si le pouvoir dans l'âge du contrôle se définit par son incorporation, et par son action à distance, alors les récits constituent un aspect de plus en plus important de cette « conduite des conduites » ou "méta-conduite» (Citton, 2010). Les récits fonctionnent alors comme un appareil particulier de capture, pour reprendre le terme de Deleuze et Guattari : elles captent l'attention en s'adressant à des désirs, à des effets et à des sensibilités qu'elles scénarisent en récits déterminés (Citton, 2010). L'intersection de ces deux dimensions constitue une sorte de "transcendance immanente»: ce qui paraît spontané et populaire, immanent aux désirs des individus, est lui-même structuré par des récits disséminés d'en haut, transcendantes à l'ordre social ${ }^{4}$. Nos réactions apparemment spontanées, nos peurs et nos désirs sont autant le produit des récits que nous consommons qu'ils en sont producteurs. La mythocratie fonctionne par une sorte de métalepse, où les valeurs et idéaux du monde fictif sont tracés sur le réel (Citton, 2010). L'asservissement machinique et l'assujettissement social apparaissent alors comme les deux faces du contrôle, deux façons d'appréhender celui-ci, qui agissent de manière très différente. Le premier opère à travers des techniques de division et de combinaison qui œuvrent en dessous de l'expérience vécue, compilant des fragments de données, et au-delà de sa conscience, recombinant ces derniers en ensembles nouveaux. Le second s'adresse nécessairement à l'individu en tant que tel, établissant le contexte et le fondement de l'expérience. Mais non moins importants sont les récits qui interpellent l'individu. Le récit de la responsabilité individuelle pour la dette a peut-être peu à voir avec le fonctionnement de celle-ci, mais il a tout à voir avec la manière dont elle est vécue. Même les tentatives pour politiser la dette («nous sommes les $99 \%$ \%) achoppent sur la honte ressentie devant l'incapacité à la rembourser (McClanahan, 2017). Les récits de la responsabilité, du travail dur, et de la capacité d'agir font partie de l'assujettissement social du contrôle. La dette est vécue comme une responsabilité individuelle, même quand elle fonctionne comme un flux impersonnel de données et de calculs.

La noo-politique et la mythocratie représentent deux politiques de postcontrôle, ellesmêmes des variations du contrôle. Chacune à sa manière prolonge la tendance à l'incorporation et à la diffusion. Dans la mesure où elles peuvent être situées par rapport à la distinction entre asservissement et assujettissement, on pourrait également dire qu'elles se différencient en termes de base et de suprastructure; la possibilité accrue d'enregistrer, de stocker et d'analyser des données est la base matérielle du contrôle, alors que la capacité accrue à produire, à disséminer et à tailler des récits est sa suprastructure. Plus précisément, elles pourraient être considérées comme un assemblage machinique du contenu et un assemblage machinique de l'expression, pour emprunter le langage de Deleuze et Guattari, ou pour simplifier, comme les corps et les esprits. Comme chez Spinoza, l'accent est mis sur le fait que les deux dimensions constituent deux façons de voir la même chose (Citton, 2017). Ce qui les relie dans ce cas, ce n'est pas, comme chez Spinoza, l'ordre infini de l'univers, mais les transformations technologiques, où l'augmentation des données et de la capacité à les traiter va de pair avec la capacité accrue à construire et à diffuser des récits qui interpellent et qui fixent les individus. La différence entre la télévision et les réseaux sociaux est que la première semble de toute évidence scénarisée, alors que les 
algorithmes qui structurent les derniers sont souvent invisibles, passant soit en dessous de l'individu au niveau du "dividuel », soit au-delà de l'individu au niveau des banques de données. Plus la structure ou le scénario semble invisible, plus ce que nous voyons, entendons et ressentons apparaît comme le produit spontané de nos désirs. Comme l'affirme Citton, l'une des caractéristiques majeures des réseaux sociaux par rapport à la télévision est l'invisibilité accrue des principes structurants de sélection et de filtrage; la télévision est toujours apparue comme le produit des réalisateurs, des producteurs et des chaînes, mais les recherches sur Google et les visionnements en ligne apparaissent comme le produit de nos désirs spontanés (Citton, 2017). Le contrôle est d'autant plus efficace quand il opère non seulement sur les conditions d'action, se distanciant de l'aspect immédiat du contact et de la contrainte, mais aussi d'une manière pratiquement indifférenciable de nos propres désirs, ou pour reprendre Deleuze, nos «motivations». Il est de plus en plus difficile de savoir où s'arrête le contrôle et où commence l'individu.

Lazzarato et Citton prolongent le concept de contrôle dans le $21^{\text {e }}$ siècle, faisant rencontrer l'essai prophétique de Deleuze et les transformations qu'il n'eût pas pu prévoir. En ce faisant, ils démontrent la tension interne du concept. Comprendre le contrôle, c'est comprendre une forme de pouvoir si immatériel, si diffus, qu'il est pratiquement impossible à détecter. Théoriser le contrôle revient alors à comprendre non seulement comment les technologies et les médias qui imprègnent notre vie quotidienne nous déterminent, mais aussi comment les désirs, les affects et les imaginaires qui constituent notre subjectivité en font partie intégrante. Dernièrement, il est important de rappeler l'affirmation de Deleuze qu'«il est facile de faire correspondre à chaque société des types de machines [...] parce qu'elles expriment les formes sociales capables de leur donner naissance et de s'en servir ». Mais bien que la technologie se présente comme un raccourci pour saisir l'extraction d'information et la personnalisation de contenus propres au contrôle, il est plus difficile de discerner les formes sociales qui agissent à travers les changements technologiques visibles. Ici, les remarques de Deleuze sur l'entreprise sont non seulement indicatives d'un fil marxiste souvent négligé dans les commentaires sur sa pensée, mais elles esquissent aussi une économie politique du contrôle. Celui-ci peut se comprendre comme la forme particulière de pouvoir correspondant à la subsomption réelle au capital, comme l'ont affirmé Hardt et Negri. Dans la subsomption réelle, le capital n'est plus imposé de l'extérieur à travers la forme-salaire et la forme-marchandise, mais refaçonne les relations du travail à ses exigences, qui tendent à incorporer toute la vie dans son orbite; ce, à mesure que la dette transforme l'éducation, la santé et le logement en sources de profit, tout en réduisant la dimension sociale du travail lui-même, et en isolant et en fragmentant les individus.

19 Comme les remarques de Deleuze l'indiquent, pointant une crise au cœur de la subsomption réelle, cette extension croissante de la portée du capital va de pair avec la recherche désespérée de profits en dehors du lieu de production. En fin de compte, c'est le paradoxe du contrôle; sa pénétration complète de la subjectivité et de l'espace social doit également être vue comme un épuisement, une diminution virtuelle du pouvoir. Rajoutant à l'observation prophétique de Deleuze, on pourrait avancer que son évocation de la dette, des services et de la production délocalisée peut se lire comme une esquisse de la désindustrialisation, d'un capital qui s'étend à toutes les sphères de la vie, car il ne peut plus générer des profits dans la sphère de la production. Son extension à la vie sociale, jusqu'aux recoins de la subjectivité, représente moins le 
mouvement d'un capital tout puissant subsumant la société entière qu'une recherche de profits jusqu'au dernier repli de relation sociale. Avec le contrôle, le capital n'a pas d'extérieur, mais il n'est rien qu'extérieur, constamment exposé au désir, à l'imagination et aux relations sociales qui sont à la fois sa condition, mais aussi sa limite. Voilà après trente ans la tâche qu'il nous reste pour théoriser le contrôle: apercevoir chaque point d'extension, y compris ceux qui pénètrent la subjectivité ellemême, comme une limite.

\section{BIBLIOGRAPHIE}

Citton, Yves (2007). « Noo-politique spinoziste? (Recension de deux livres récents sur Spinoza, de Lorenzo Vinciguerra et de Pascal Sévérac) », Multitudes, 27 (hiver) : <http://

multitudes.samizdat.net/Noo-politique-spinoziste-Recension>. [consulté 20 jan. 2019].

- (2010). Mythocratie : Storytelling et Imaginaire de Gauche. Paris : Éditions Amsterdam.

- (2014). (dir.) L'Economie de l'attention. Nouvel horizon du capitalisme, Paris : La Découverte.

Deleuze, Gilles (1990). "Post-scriptum sur les sociétés de contrôle" in Pourparlers, Paris : Minuit.

Deleuze, Gilles et Félix Guattari (1972). L'Anti-đEdipe. Capitalisme et schizophrénie 1, Paris : Minuit.

- (1980). Mille plateaux. Capitalisme et schizophrénie 2, Paris : Minuit.

Foucault, Michel (1993) (1975). Surveiller et punir, Paris : Gallimard.

Hardt, Michael and Antonio Negri (1994). The Labor of Dionysus: A Critique of the State Form. Minneapolis : University of Minnesota Press.

- (2012). Declaration. (Distribué par Argo Navis Author Services (autoédition)).

Lazzarato, Maurizio (2004). Les Révolutions du Capitalisme. Paris : Les Empêcheurs de Penser en Rond.

- (2010). La Fabrique de l'homme endetté. Essai sur la condition néolibérale, Paris : Editions Amsterdam.

- (2014). Signs and Machines: Capitalism and the Production of Subjectivity (trans. Joshua David Jordan). New York : Semiotext(e).

Lordon, Frédéric (2012). «Derrière l'idéologie de la légitimité, la puissance de la multitude : le traité politique comme théorie générale des institutions sociales ", La Multitude Libre: Nouvelles lectures du Traité Politique. Paris : Éditions Amsterdam, 105-30.

Marx, Karl (1932) [1844]. « Comment on James Mill. Éléments d'économie politique », Marxist.org : <https://www.marxists.org/archive/ marx/works/1844/james-mill/index.htm>. [consulté 20 jan. 2019]

McClanahan, Annie (2017). Dead Pledges: Debt, Crisis, and Twenty-First Century Culture. Stanford : Stanford University Press. 


\section{NOTES}

1. Ce passage et les pages attenantes sont omis dans la traduction française de ce texte par Roger Dangeville (dans Marx, Engels, Les Utopistes, Maspero, 1976, disponible sur le site <classiques.uqac.ca>). Il s'agit ici d'une retraduction - à considérer comme provisoire - à partir de l'anglais, en attendant la version française intégrale de l'allemand original, projet annoncé en 2019 par une équipe basée à l'université de Nantes (NdT).

2. Comme exemple, citons le petit coup de griffe de Foucault dans Surveiller et Punir: "Notre société n'est pas celle du spectacle, mais de la surveillance : sous la surface des images, on investit les corps en profondeur; derrière la grande abstraction de l'échange, se poursuit le dressage minutieux et concret des forces utiles ; les circuits de la communication sont les supports d'un cumul et d'une centralisation du savoir...» $(1975: 252)$. L'étude du pouvoir et des corps s'oppose ici à l'étude des médias, des images et de la communication. C'est justement cette opposition que les théoriciens contemporains du contrôle cherchent à surmonter.

3. Dans une recension pour la revue Multitudes, Citton reprend la question de la «noo-politique » avancée par Lazzarato, affirmant que la pensée de Spinoza rend possible une noo-politique qui fonctionne sur les affects, et par la capture du désir (2007:np).

4. Citton tire sa notion de "transcendance immanente" de l'interprétation de Spinoza par Frédéric Lordon. D'après Lordon, étant donné que les affects, et avec eux les désirs, sont renforcés par d'autres, par ce que Spinoza appelle l'imitation des affects, et que les gens sont pour la plupart ignorants des causes de leurs affects, les conditions sont créées dans lesquelles les individus ne reconnaissent pas la cause de leurs comportements affectifs, acceptant comme naturel et spontané ce qui est institué (2008: 126). La transcendance immanente est une réponse à la question posée par Spinoza, et reprise par Deleuze et Guattari : "pourquoi les hommes combattent-ils pour leur servitude comme s'il s'agissait de leur salut?»

\section{AUTEURS}

\section{JASON READ}

Professeur de philosophie à l'University of Southern Maine. 